\title{
Extracting Full-Field Dynamic Strain on a Wind Turbine Rotor Subjected to Arbitrary Excitations using 3D Point Tracking and a Modal Expansion Technique
}

\author{
Javad Baqersad $^{1}$, Christopher Niezrecki ${ }^{2}$, Peter Avitabile ${ }^{3}$ \\ University of Massachusetts Lowell \\ One University Ave, Lowell, MA 01854
}

\begin{abstract}
Health monitoring of rotating structures such as wind turbines and helicopter rotors is generally performed using conventional sensors that provide a limited set of data at discrete locations near or on the hub. These sensors usually provide no data on the blades or inside them where failures might occur. Within this paper, an approach was used to extract the full-field dynamic strain on a wind turbine assembly subject to arbitrary loading conditions. A three-bladed wind turbine having 2.3-meter long blades was placed in a semi-built-in boundary condition using a hub, a machining chuck, and a steel block. For three different test cases, the turbine was excited using 1) pluck testing, 2) random impacts on blades with three impact hammers, and 3) random excitation by a mechanical shaker. The response of the structure to the excitations was measured using three-dimensional point tracking. A pair of high-speed cameras was used to measure displacement of optical targets on the structure when the blades were vibrating. The measured displacements at discrete locations were expanded and applied to the finite element model of the structure to extract the full-field dynamic strain. The results of the paper show an excellent correlation between the strain predicted using the proposed approach and the strain measured with strain-gages for each of the three loading conditions. The approach used in this paper to predict the strain showed higher accuracy than the digital image correlation technique. The new expansion approach is able to extract dynamic strain all over the entire structure, even inside the structure beyond the line of sight of the measurement system. Because the method is based on a non-contacting measurement approach, it can be readily applied to a variety of structures having different boundary and operating conditions, including rotating blades.
\end{abstract}

Keywords: Modal expansion, full-field strain, wind turbine, health monitoring, blade, response identification.

\footnotetext{
${ }^{1}$ Javad_Baqersad@uml.edu; University of Massachusetts Lowell, One University Ave Lowell, MA 01854. Tell: +1-978-761-5572.

${ }^{2}$ Christophoer_Niezrecki@uml.edu; University of Massachusetts Lowell, One University Ave, Lowell, MA 01854.

${ }^{3}$ Peter_Avitabile@uml.edu; University of Massachusetts Lowell, One University Ave Lowell, MA 01854.
} 


\section{NOMENCLATURE}

$\begin{array}{ll}\mathbf{E R T O}_{\mathbf{n}} & \text { expanded real-time operating data } \\ \mathbf{K}_{\mathbf{a}} & \text { reduced stiffness matrix } \\ \mathbf{K}_{\mathbf{n}} & \text { full-space stiffness matrix } \\ \mathbf{M}_{\mathbf{a}} & \text { reduced mass matrix } \\ \mathbf{M}_{\mathbf{n}} & \text { full-space mass matrix } \\ \mathbf{P} & \text { mode contribution vector } \\ \mathbf{R T O} \mathbf{O}_{\mathbf{a}} & \text { real-time operating data at reduced-space } \\ \mathbf{R T O} \mathbf{O}_{\mathbf{i}} & \text { displacement time traces for point } i \\ \mathbf{R T O} \mathbf{O}_{\mathbf{j}} & \text { displacement time traces for point } j \\ \mathbf{T} & \text { transformation matrix } \\ \mathbf{U}_{\mathbf{a}} & \text { reduced mode shape matrix } \\ \mathbf{U}_{\mathbf{a}} & \text { generalized inverse of reduced shape matrix } \\ \mathbf{U}_{\mathbf{d}} & \text { mode shape matrix at deleted degrees of freedom } \\ \mathbf{U}_{\mathbf{n}} & \text { full-space mode shape matrix } \\ \mathbf{X}_{\mathbf{a}} & \text { reduced-space displacement vector } \\ \mathbf{X}_{\mathbf{d}} & \text { displacement vector at deleted degrees of freedom } \\ \mathbf{X}_{\mathbf{i}} & \text { displacement vector } i \\ \mathbf{X}_{\mathbf{j}} & \text { displacement vector } j \\ \mathbf{X}_{\mathbf{n}} & \text { full-space displacement vector }\end{array}$




\section{INTRODUCTION}

Wind power has recently emerged as one of the major sources of clean renewable energy. Modern horizontal axis wind turbines are growing in size and number in order to meet increasing energy demands. The escalating initial capital investment of the wind turbines has generated wide interest in damage prognosis and health monitoring of these large structures. Traditional sensors such as accelerometers and strain-gages can only measure at discrete locations. Wireless sensors have great potential to be implemented in rotating blades; however, these sensors can suffer from EMI and data transmission challenges and require a significant number to fully cover an area of interest. For wind turbines, most of the sensors used for monitoring are located on the nacelle to monitor the gearbox, rotor, and generator, and there are few to no sensors mounted to the turbine blades where failure might occur. At the 2014 Sandia Wind Turbine Blade Workshop, it was identified that the edgewise-resonant vortex induced vibration of wind turbines and its effect on blades is currently poorly understood primarily because of a lack of appropriate measurement tools to quantify its influence and effect [1]. Therefore, a health monitoring algorithm for the rotating structures that has a distributed sensing capability over the entire turbine blade is desirable. Such an algorithm not only can help predict failure and remaining fatigue life of the structure but also can improve the understanding of loading conditions that these structures experience.

In this paper, an algorithm is proposed to extract dynamic strain on rotating structures using a non-contact measurement approach and finite element (FE) modeling. The proposed method uses three-dimensional point tracking along with a modal expansion technique. Therefore, these two techniques are now reviewed.

Three-dimensional (3D) digital image correlation (DIC) and three-dimensional point tracking (3DPT) are both non-contacting measurement approaches that analyze stereo image pairs to extract the 3D motion of surface pattern or specific points. The 3D DIC technique is performed using a patterned area of interest and can identify the full-field dynamic displacement and strain over the visible surface of the structure. For 3DPT, high contrast optical targets are mounted to the structure and the displacements of those targets are measured.

Recently, researchers have started to use the stereophotogrammetry technique to measure dynamics of transient phenomena [2,3] and vibrations [4-6] in structures. The technique has been successfully applied to non-rotating wind turbine blades to measure displacement vectors and surface strain [7-10]. More recently, researchers have tried to use the stereophotogrammetry technique on rotating structures. Helfrick et al. [11] used low speed cameras to perform a DIC measurement on a small rotating fan. In an effort to minimize measurement noise from DIC data, Wang et al. [12] used an image decomposition approach and extracted frequency response functions for several structures. These results were used to extract shape features [13] and eventually update finite element models $[14,15]$. Due to the difficulties regarding surface preparations and high camera resolution needed for DIC measurements, it is far less common to find published literature that 
uses DIC system for rotating structures. On the other hand, the 3DPT technique has more frequently been applied to the dynamic measurement of rotating structures. This technique has been used for measuring displacements of optical targets on small-scale wind turbines $[16,17]$ as well as utility-scale wind turbines [18]. The 3DPT technique has also been successfully used to measure the dynamics of helicopter rotors [1922]. Although measuring displacements of discrete targets mounted on the blades of rotating structures could improve the physical understanding of rotating structures, these measurements by themselves cannot provide detailed information about the levels of strain that structure sees, which is important for some health monitoring algorithms. Furthermore, traditional sensors (e.g. strain gages and accelerometers) can only measure strain at discrete locations. However, the location that a crack initiates might be different from the place where that sensor has been installed. Thus, conventional point wise sensors might not capture the deformations or strains at critical locations in the vicinity of the sensors. Using full-field predicted strain based upon the measured optical data may help to find the critical areas on the surface or within the internal parts of the structure. For example if an excessive loading event generates a high strain (or stress) it may be possible to monitor these events at all points on the structure to assess possible damage or estimate fatigue accumulation, thereby using such an approach to perform structural health monitoring. Therefore, establishing a comprehensive algorithm that enables full-field monitoring of structures using limited set of measurements is desirable.

Researchers have used Inverse Finite Element Method and expansion algorithms to extract full-field response of a structure using limited measurements. Inverse Finite Element Method has generally been used for extracting full-field response of the structure using measured strain [23-25]. This method can be used for both static and dynamic loading conditions but the application of this approach for complicated structures have not been examined.

Modal reductions/expansion techniques have conventionally been used for correlation and validation purposes. These techniques are usually used to reduce a numerical model and compare it to the experimental model at a limited number of measured data points. On the other hand, these techniques can expand the measured mode shapes to compare them to the numerical model at a full-space using a transformation matrix. The transformation matrix can be one of several commonly used matrices in an expansion algorithm including Guyan Condensation [26], Dynamic Condensation [27], Improved Reduced System [28], and SEREP (System Equivalent Reduction and Expansion Process) [29], but because SEREP can produce an exact reduced system, it is used for all the work presented herein.

The modal expansion techniques can be used to expand real-time measured data. Chipman et al. [30] showed that by expanding the limited set of data points measured with a laser Doppler vibrometer, an improved description of the real time operating response can be extracted. Pingle et al. [31] further expanded the method and applied it on several academic structures. Carr et al. [32] used the method to expand out-of-plane 
data for an aluminum beam and a blade placed in a cantilevered configuration and excited using a mechanical shaker. In their work, the loading condition was controlled by only applying either single or dual-sinusoidal inputs and may not necessarily replicate the operating condition of the structure. Iliopoulos et al. [33] expanded limited set of data measured with accelerometers and applied it to an analytical beam model to predict dynamic strain on a wind turbine tower. Although SEREP only uses linear mode shapes, it has also been successfully used to expand dynamic strain for numerical models of structures that include small nonlinearities [34, 35]. Using strain mode shapes is another approach that has been used by researchers to extract full-filed displacement and strain using strain measured at limited locations $[36,37]$.

Using iterative methods can improve the accuracy of the predicted dynamic response. Chierichetti et al. [3840] used a confluence algorithm to extract the full-field dynamic response of several structures by using limited set of measured data. In this approach, an initial guess of the load is applied to the numerical model and the response of the structure due to the initial load is extracted. By comparing the numerical response of the structure at the measurement points to the experimentally measured results, the applied force is updated; the updated force is then applied to the numerical model and the response is compared at sensor locations. This procedure continues until the difference between the measured response and predicted response with the numerical model is below a tolerance level. Using iterative method can make corrections for small nonlinearities. In another work by Chierichetti et al. [41] the experimental modal model of a system was used to expand the measured data and obtain the response for unmeasured degrees of freedom.

The current paper uses photogrammetry in conjunction with an expansion methodology to extract the dynamic strain on a commercial wind turbine consisted of several components. In order to demonstrate that the method works for different operating conditions, several types of loading (transient pluck test, multiple simultaneous impact, and random) were used to excite the structure. The results of this paper can be applied to operating wind turbines or helicopter rotors. Being able to experimentally, fully characterize the global mechanics of these structures is not currently possible and highly desirable for model validation, condition based monitoring, and fatigue life prediction. Using such an approach is also beneficial to help assess the loading that helicopter rotors and wind turbine blades experience during operation; measurement of these loads is not currently possible. The proposed approach will help to obtain a better understanding of the influence of aerodynamic loads on wind turbine blades. Another application resulting from this work will be the ability to determine the actual strain throughout a structure at locations where there are no sensors mounted, including internal surfaces where it is difficult or not practical to attach strain gages. This paper represents the first work to predict the full-field strain on a three-bladed turbine for a variety of realistic excitations and to validate the measurements with instrumented strain gages. The theoretical basis for this work is now reviewed. 


\section{THEORY}

\subsection{Expansion}

Model reduction/expansion approaches can be used to augment measurements and extract data for unmeasured degrees of freedom (DOF). In the current work, the modal expansion is used to augment the real time operating data at optical targets and extract the full-field dynamic displacement at all finite element degrees of freedom. For all modal expansion algorithms, the relationship between the full-space and reducedspace model can be written as:

$$
\mathbf{X}_{\mathbf{n}}=\mathbf{T} \mathbf{X}_{\mathbf{a}}
$$

where $\mathbf{X}_{\mathbf{n}}$ and $\mathbf{X}_{\mathbf{a}}$ represent the displacement vectors in full-space and reduced-space, respectively. The transformation matrix $\mathbf{T}$ is used to project the full-space mass and stiffness matrices to the reduced-space. The full-space matrices can be related to reduced-space matrices using the following equations.

$$
\begin{aligned}
& M_{a}=T^{T} M_{n} T \\
& K_{a}=T^{T} K_{n} T
\end{aligned}
$$

where $\mathbf{M}_{\mathbf{n}}$ and $\mathbf{K}_{\mathbf{n}}$ represent mass and stiffness matrices in the full-space and $\mathbf{M}_{\mathbf{a}}$ and $\mathbf{K}_{\mathbf{a}}$ are in the reducedspace, respectively. The SEREP transformation matrix is developed by using mode shapes of the system at the full-space. Based on modal theory, the response of a structure can be expressed by a linear combination of the modes of that structure. This theory can be written as:

$$
\mathbf{X}_{\mathbf{n}}=\mathbf{U}_{\mathbf{n}} \mathbf{P}
$$

where $\mathbf{U}_{\mathbf{n}}$ denotes the mode shapes of the structure at full-space and $\mathbf{P}$ represents the contribution of each mode in the response of the structure. Equation (4) can be written as:

$$
\mathbf{X}_{\mathbf{n}}=\left[\begin{array}{ll}
\mathbf{X}_{\mathbf{a}} & \mathbf{X}_{\mathbf{d}}
\end{array}\right]=\left[\begin{array}{ll}
\mathbf{U}_{\mathbf{a}} & \mathbf{U}_{\mathbf{d}}
\end{array}\right] \mathbf{P}
$$

where $\mathbf{U}_{\mathbf{a}}$ and $\mathbf{U}_{\mathbf{d}}$ are the mode shapes at the active and deleted DOF, respectively. Writing Equation (5) in terms of active DOF gives:

$$
\mathbf{X}_{\mathbf{a}}=\mathbf{U}_{\mathbf{a}} \mathbf{P}
$$

In this equation, $\mathbf{U}_{\mathbf{a}}$ is not a square matrix (the number of equations is not equal to the number of unknowns). Thus, the Equation was solved using a generalized inverse method. Pre-multiplying both sides of Equation 6 by $\left(\mathbf{U}_{\mathbf{a}}{ }^{\mathrm{T}} \mathbf{U}_{\mathbf{a}}\right)^{-1} \mathbf{U}_{\mathbf{a}}{ }^{\mathrm{T}}$ gives:

$$
\begin{aligned}
& \left(\mathbf{U}_{\mathbf{a}}^{\mathrm{T}} \mathbf{U}_{\mathrm{a}}\right)^{-\mathbf{1}} \mathbf{U}_{\mathbf{a}}^{\mathrm{T}} \mathbf{X}_{\mathrm{a}}=\left(\mathbf{U}_{\mathbf{a}}^{\mathrm{T}} \mathbf{U}_{\mathbf{a}}\right)^{-1} \mathbf{U}_{\mathbf{a}}^{\mathrm{T}} \mathbf{U}_{\mathbf{a}} \mathbf{P} \\
& \mathbf{P}=\left(\mathbf{U}_{\mathbf{a}}^{\mathbf{T}} \mathbf{U}_{\mathbf{a}}\right)^{-\mathbf{1}} \mathbf{U}_{\mathbf{a}}^{\mathbf{T}} \mathbf{X}_{\mathbf{a}} \\
& \mathbf{P}=\mathbf{U}_{\mathbf{a}}{ }^{\mathbf{g}} \mathbf{X}_{\mathbf{a}} \\
& \mathbf{X}_{\mathbf{n}}=\mathbf{U}_{\mathbf{n}} \mathbf{U}_{\mathbf{a}}{ }^{\mathbf{g}} \mathbf{X}_{\mathbf{a}}
\end{aligned}
$$


Thus, the SEREP transformation is written as:

$$
\mathbf{T}=\mathbf{U}_{\mathbf{n}} \mathbf{U}_{\mathbf{a}}{ }^{\mathbf{g}}
$$

This matrix can also be expressed as:

$$
\mathbf{T}=\mathbf{U}_{\mathbf{n}}\left(\mathbf{U}_{\mathbf{a}}{ }^{\mathbf{T}} \mathbf{U}_{\mathbf{a}}\right)^{-\mathbf{1}} \mathbf{U}_{\mathbf{a}}{ }^{\mathbf{T}}
$$

The mode reduced by this method yields the exact frequencies and mode shapes as those obtained from the eigensolution of the full size matrices.

For the current work, the SEREP transformation matrix was developed using the finite element mode shapes. However, it can be developed using measured modal vectors such as the modes measured with Laser Doppler vibrometer in Chipman et al.'s work [30]. Equation 1 is used to expand measured real-time operating data RTO $_{\mathbf{a}}$ to the full-space of the finite element model $\mathbf{E R T O}_{\mathbf{n}}$ and is written as:

$$
\text { ERTO }_{\mathbf{n}}=\text { T RTO }_{\mathbf{a}}
$$

\subsection{Correlation Tools}

The modal assurance criterion (MAC) [42] is a tool for quantifying the correlation between two vectors at all degrees of freedom. The MAC is given by:

$$
\operatorname{MAC}_{i j}=\frac{\left(\mathbf{X}_{i}^{\mathrm{T}} \mathbf{X}_{j}\right)^{2}}{\left(\mathbf{X}_{\mathbf{i}}^{\mathbf{T}} \mathbf{X}_{\mathbf{i}}\right)\left(\mathbf{X}_{\mathbf{j}}^{\mathbf{T}} \mathbf{X}_{\mathbf{j}}\right)}
$$

where $\mathbf{X}_{\mathbf{i}}$ and $\mathbf{X}_{\mathbf{j}}$ are displacement vectors (usually mode of the structure). The MAC values close to 1.0 indicate strong similarity between vectors, where values close to 0.0 indicate minimal or no similarity. The TRAC is used in the current paper to find the correlation between two time traces. The TRAC can be written as:

$$
\text { TRAC }_{i j}=\frac{\left(\text { RTO }_{i}{ }^{\text {T }} \text { RTO }_{j}\right)^{2}}{\left(\text { RTO }_{i}{ }^{\text {T }} \text { RTO }_{i}\right)\left(\text { RTO }_{\mathbf{j}}{ }^{\text {T }} \text { RTO }_{j}\right)}
$$

where $\mathbf{R T O} \mathbf{O}_{\mathbf{i}}$ and $\mathbf{R T O} \mathbf{O}_{\mathbf{j}}$ represent time traces for point $\mathrm{i}$ and $\mathrm{j}$. It should be noted that the TRAC only consider the correlation between the traces and not their amplitudes. Thus, if a time traces is scaled and compared to the original signal, the TRAC shows a strong correlation although the amplitudes of the traces are not equivalent.

Similar to MAC, TRAC values close to 1.0 indicate strong similarities, where values close to 0.0 indicate minimal or no similarity. The MAC and TRAC values in this paper are presented in percent (0 to 100\%). 


\section{TEST SETUP}

A Skystream 4.7 wind turbine with 2.3-meter blades was used as the test structure. In order to provide a configuration that mimics the operating condition of a wind turbine, three blades of Skystream 4.7 were assembled to a hub and were fixed with a machining chuck which was rigidly bolted to a 500-lb block as shown in Figure 1.

A pair of Photron high-speed cameras was focused and calibrated using a 2-meter calibration cross. The calibration deviation for this test was calculated to be 0.0225 pixels. According to the PONTOS ${ }^{\mathrm{TM}}$ user manual [43], for a good calibration, the calibration deviation needs to be less than or equal to 0.04 pixels. The 4 mega-pixel cameras equipped with 24-mm lenses were mounted to a 2-meter aluminum bar and placed approximately 5.6-meter away from the turbine hub so they can observe the whole turbine area (see Figure 1). Proper illumination was provided using several lights. A series of optical targets $(\sim 79)$ were mounted to the blades and the fixture to measure the in-plane and out-of-plane displacements of all the components. The cameras were configured to record images at a rate of $125 \mathrm{fps}$.
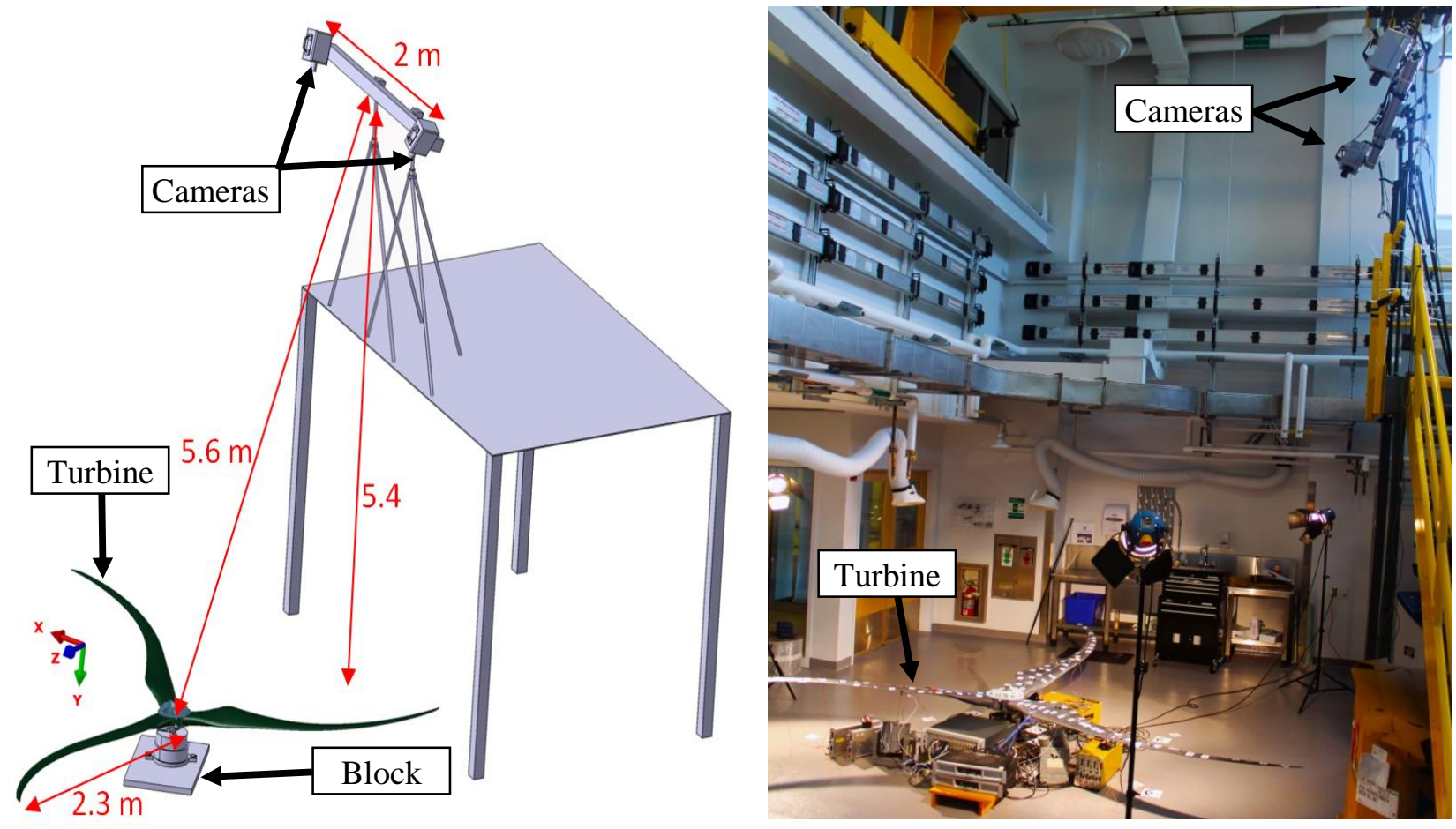

Figure 1. A schematic of the set up of the cameras and turbine (left) and a photo of the actual test setup (right). 


\section{MODELING AND DATA PROCESSING}

A finite element (FE) model of the blade and components including the hub, plate, shaft, and the fixture was developed in ABAQUS. The blades, hub, and other flexible components were modeled using quadratic tetrahedral elements (ABAQUS C3D10). These elements are not very sensitive to mesh density, require far fewer elements to converge to a solution compared to linear elements, and have shown to produce accurate results for the eigensolution of blades [44]. The flexible modes of the fixture occur at higher frequencies (the first flexible mode of the block is about $300 \mathrm{~Hz}$ ) than the turbine modes (the first mode of the turbine is at 5.3 $\mathrm{Hz}$ ). Therefore, the fixture (the block and chuck) was modeled using rigid elements and proper weight and inertial moment were applied to the model. After modeling the components, they were attached using rigid tie elements, which connect all degrees of freedom of the components at the contact surface. The blades are tightly secured in the hub using 12 bolts; thus using tie elements can replicate this secure connection. The connection between the fixture and the ground (layers of foam) was modeled using linear springs. In order to validate the FE mode shapes, an experimental modal test was performed on the wind turbine in a semi-builtin configuration. In this test, the turbine was excited using three mechanical shakers and the response of the structure was measured at 36 points using triaxial accelerometers. A more detailed description of the the experimental modal test can be found in [45]. A comparison between the FE and experimental mode shapes was performed using the modal assurance correlation. The constants of the springs (modeling the layers of foam) were updated using experimental results. After updating the model, strong similarities could be seen between the FE and test models for most of the modes as shown in Figure 2. It should be noted that the modes that do not show strong correlations are generally related to the shapes that were generated due to the fixture modes (e.g. modes 4 and 5). 


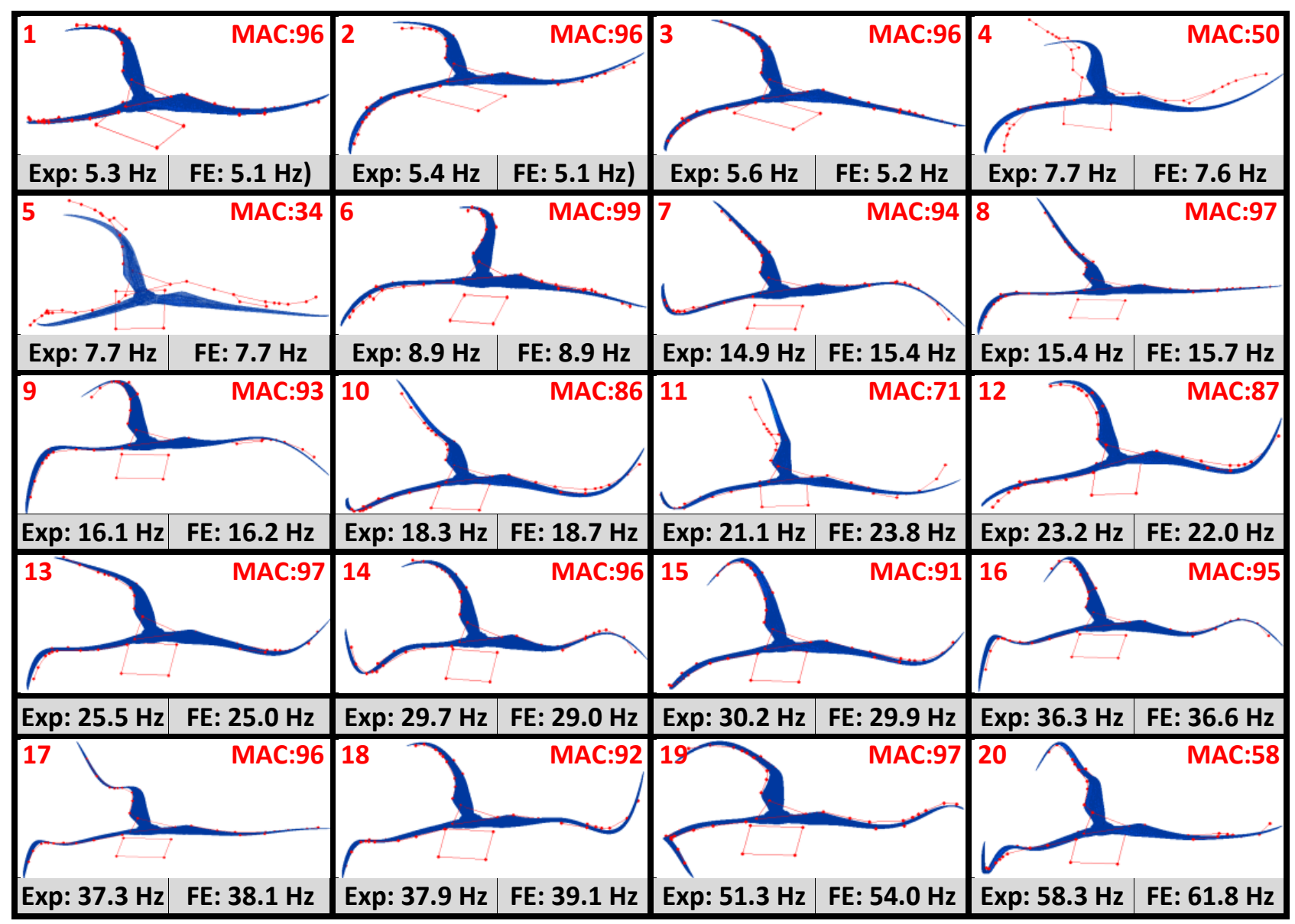

Figure 2. The first twenty FE mode shapes of the turbine correlated with experimentally measured modes shapes using the Modal Assurance Criterion.

A flowchart of the proposed process for dynamic strain extraction along with the comparison that was performed in the current paper is shown in Figure 3. As can be seen, the test rig included the blades and the hub that were attached to the fixture. Furthermore, eight strain-gages were installed on the blades to measure dynamic strain at discrete locations. Strain measured with strain-gages was used for validation purposes.

Figure 3 also shows an overview of the proposed technique for dynamic strain extraction. A pair of stereophotogrammetric cameras was used to measure dynamic displacement of optical targets mounted on the structure. After extracting the dynamic displacement of optical targets by using the three-dimensional point tracking technique, the limited set of measured displacements were expanded to all FE degrees of freedom using the FE mode shapes. The calculated displacements were then applied to the FE model to extract the dynamic strain (or stress) at each stage. 
The number of modes that are used for the expansion depends on the modes that are excited during operation. In fact, the number of modes that are used in the model should be able to span the frequency range of the modes excited during operation. For example for a sinusoidal excitation at a resonant frequency of the turbine, using only the corresponding mode would be sufficient to expand the real time operating data. However, for a more complicated loading condition, using more modes for the expansion process is necessary. Because the arbitrary excitations used in this study may excite many modes, the first 20 modes of the structure were used to expand the measured displacement. Using that many modes assures that the expansion process will be able to cover the deformations that might occur in a frequency bandwidth from 0 to $\sim 60 \mathrm{~Hz}$.
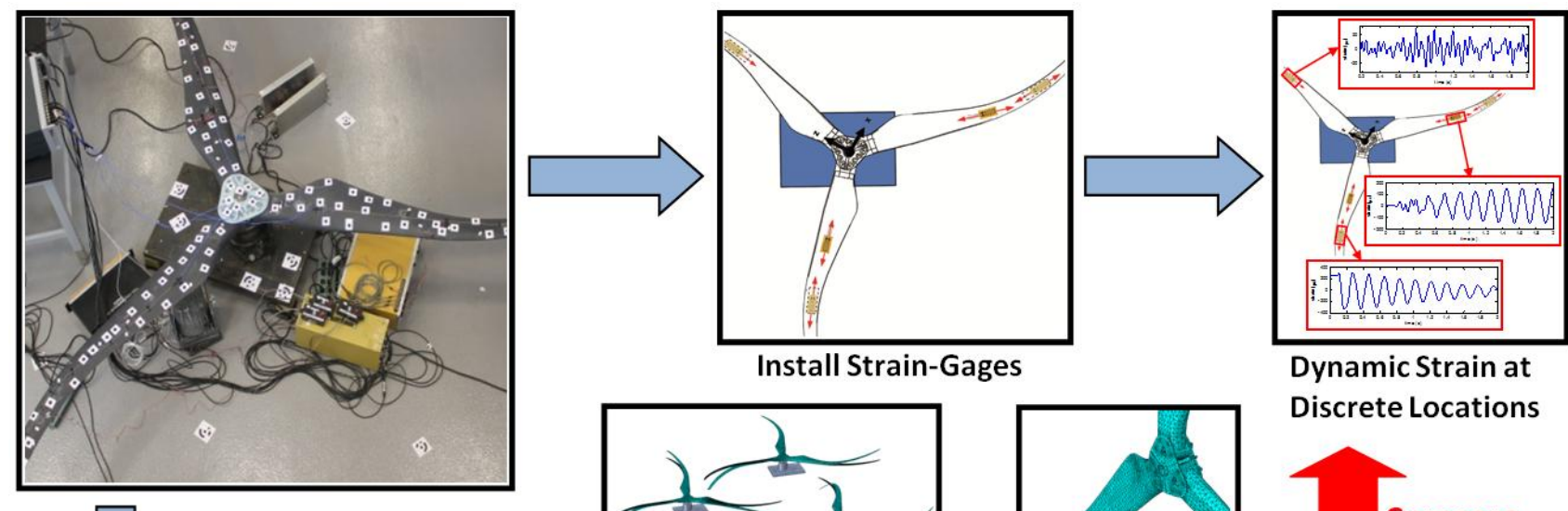

Install Strain-Gages

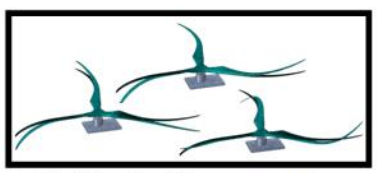

FE Mode Shapes
Dynamic Strain at Discrete Locations

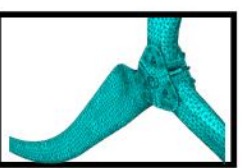

FE Model

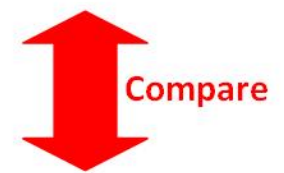

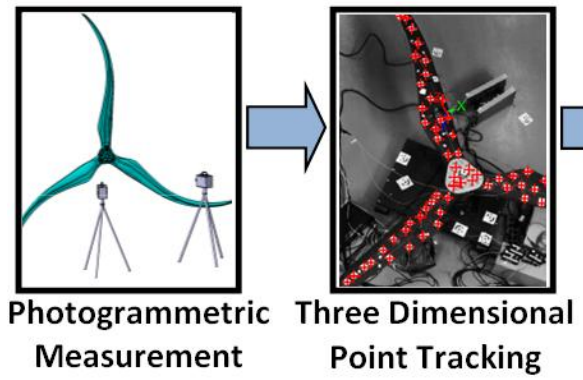

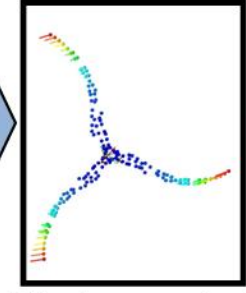

Displacement at Limited Locations

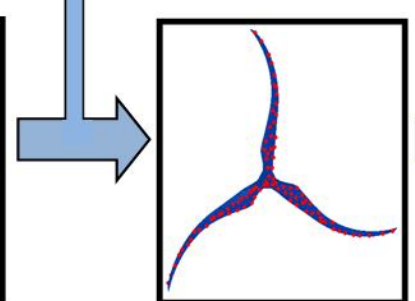

Full Nodal Displacement

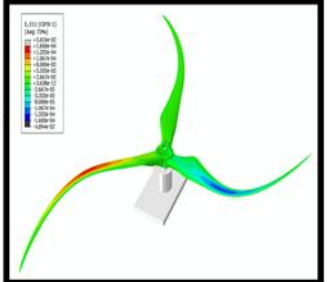

Full-Field Dynamic Strain

Figure 3. A flowchart showing the dynamic strain measurement using strain-gages (upper) and proposed approach (lower).

It should be noted that the SEREP not only expands the measurement points but also smoothes the measured data. On the other hand, by using SEREP as the expansion method, if there is some noise on the measured data, the expansion process minimizes the noise to fit the measured displacement with the mode shapes of the system. In order to illustrate the influence of the SEREP process on the measured data, the measured displacements of the optical targets before and after expansion are shown in Figure 4. In this plot, the $\mathrm{x}$-axis 
shows the locations of targets with respect to the center and the y-axis shows the displacement of optical targets at a sample in time. As can be seen, the SEREP did not necessarily preserve all the measured points as they were measured; in fact, this process is a least square curve-fitting method.

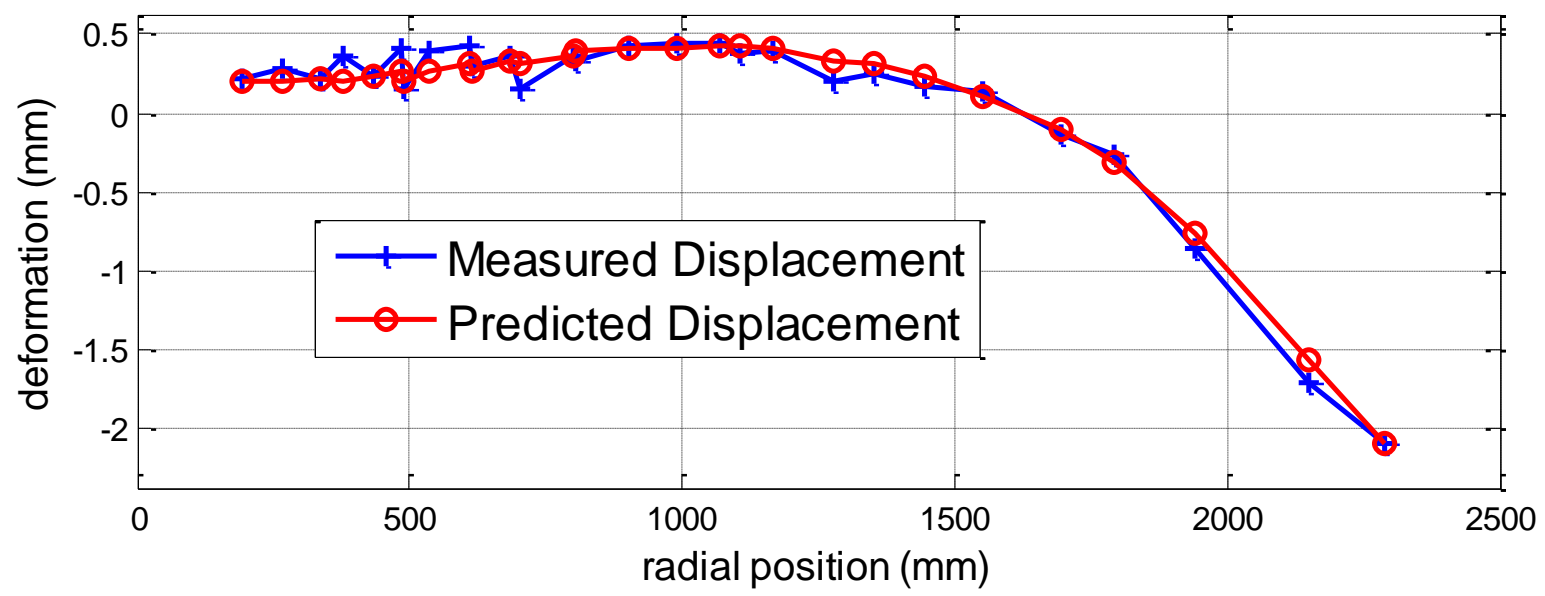

Figure 4. Comparing measured displacements of optical targets and predicted displacements (after passing through the SEREP) for the out of plane deformations of one of the blades.

\section{CASES STUDIED}

The wind turbine may experience different loading conditions during operation. In order to consider all possible conditions that a wind turbine may experience, different loading scenarios were examined in this paper. These loading scenarios include a pluck testing, arbitrary impact on all three blades, and random excitation with a shaker. The description of the loading scenarios, the work carried out for each case, and the results are now reviewed.

\subsection{Pluck Test}

In this test, the tip of a single blade was displaced using a dead-weight. As shown in Figure 5, the weight imposed initial displacement on the turbine blade that the mass was attached to. By cutting the connecting string, the wind turbine started to freely oscillate and the dynamic displacements of optical targets were measured during this oscillation using three-dimensional point tracking. 


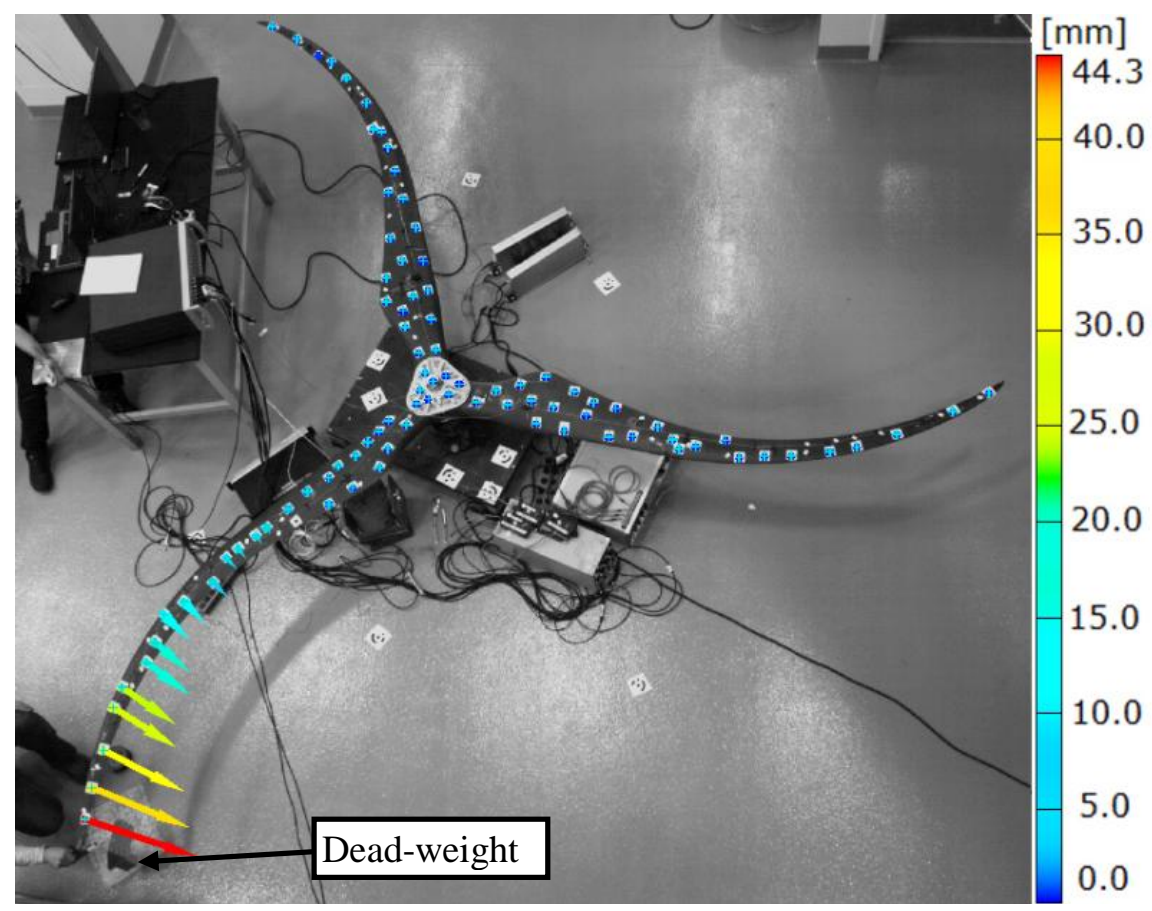

Figure 5. A photo of the test set-up showing the dead-weight hung from the lower left blade and the associated displacement vectors of the optical targets.

In order to extract the full-field dynamic strain on the structure, measured displacements of the optical targets were expanded and applied to the FE model. Therefore, the full-field dynamic strain was extracted on the structure (see Figure 6). The predicted strain was compared to the measured strain at strain-gage locations to validate the technique. As shown in Figure 6, the predicted strain is in close agreement with the measured strain. It can be seen in Figure 6 that the strain is initially higher in the blade that the dead weight was attached to. After several oscillations, the two other blades also started to oscillate and approach a high level of strain. 


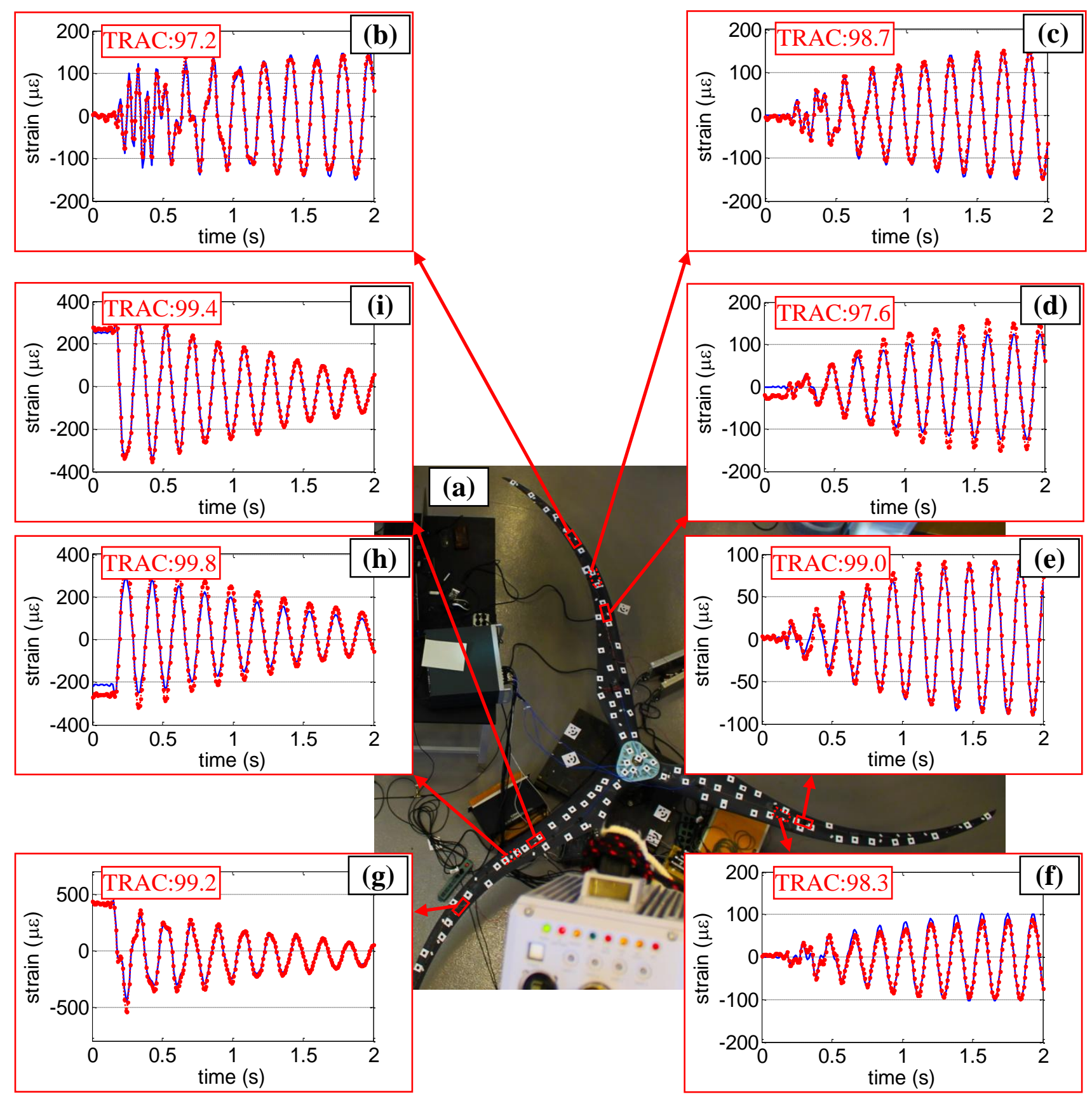

Figure 6. Comparison of the strain measured with mounted strain-gages ( $\square$ ) and the predicted strain using the proposed approach $(:)$ during the pluck test. (a) The photo shows the location of the straingages (red boxes), where the comparison is performed. Comparison at location of sensors (b) $S 11$, (c) $S 12$, (d) $S 13$, (e) $S 21$, (f) $S 22$, (g) $S 31$, (h) $S 32$, and (i) $S 33$. It is important to note that sensors $S 12$, $S 22$, and $S 32$ were mounted to the underside surface of the blades where no optical targets were placed or measured. 


\subsection{Random Impact Excitations}

In the second loading scenario, a highly arbitrary loading condition was considered. In this case, three impact hammers were used to simultaneously make random impact excitations that were spatially distributed along the three blades (see Figure 7). This impacting input was completely random and impulsive and could replicate a very complicated loading condition for wind turbines.

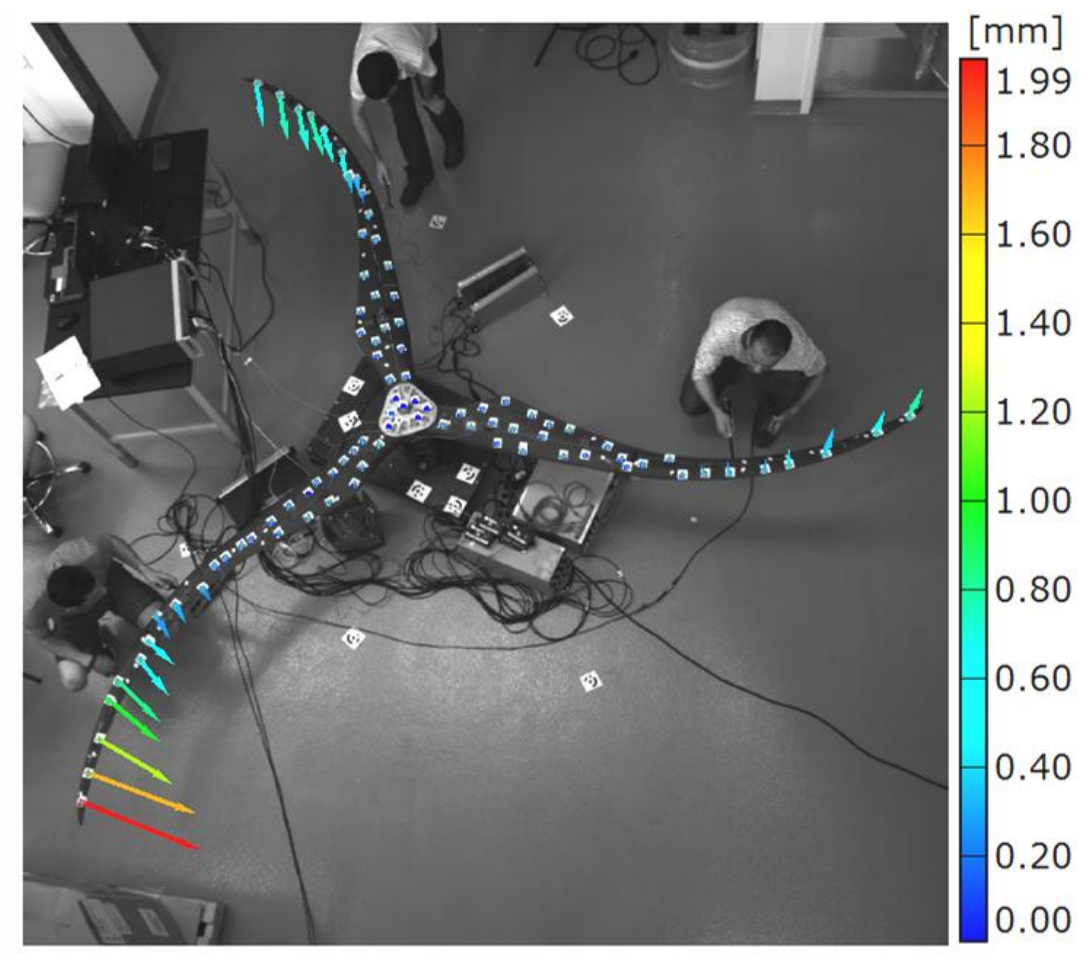

Figure 7. A photo of the test set-up showing arbitrary impacts made on the three blades using three impact hammers and the associated displacement vectors of optical targets due to the impacts at one instant in time.

The measured strain using strain-gages for the random impact excitations is compared to predicted strain in Figure 8 . The figure shows that although many modes were excited during this testing, the proposed approach could predict the dynamic strain accurately. 


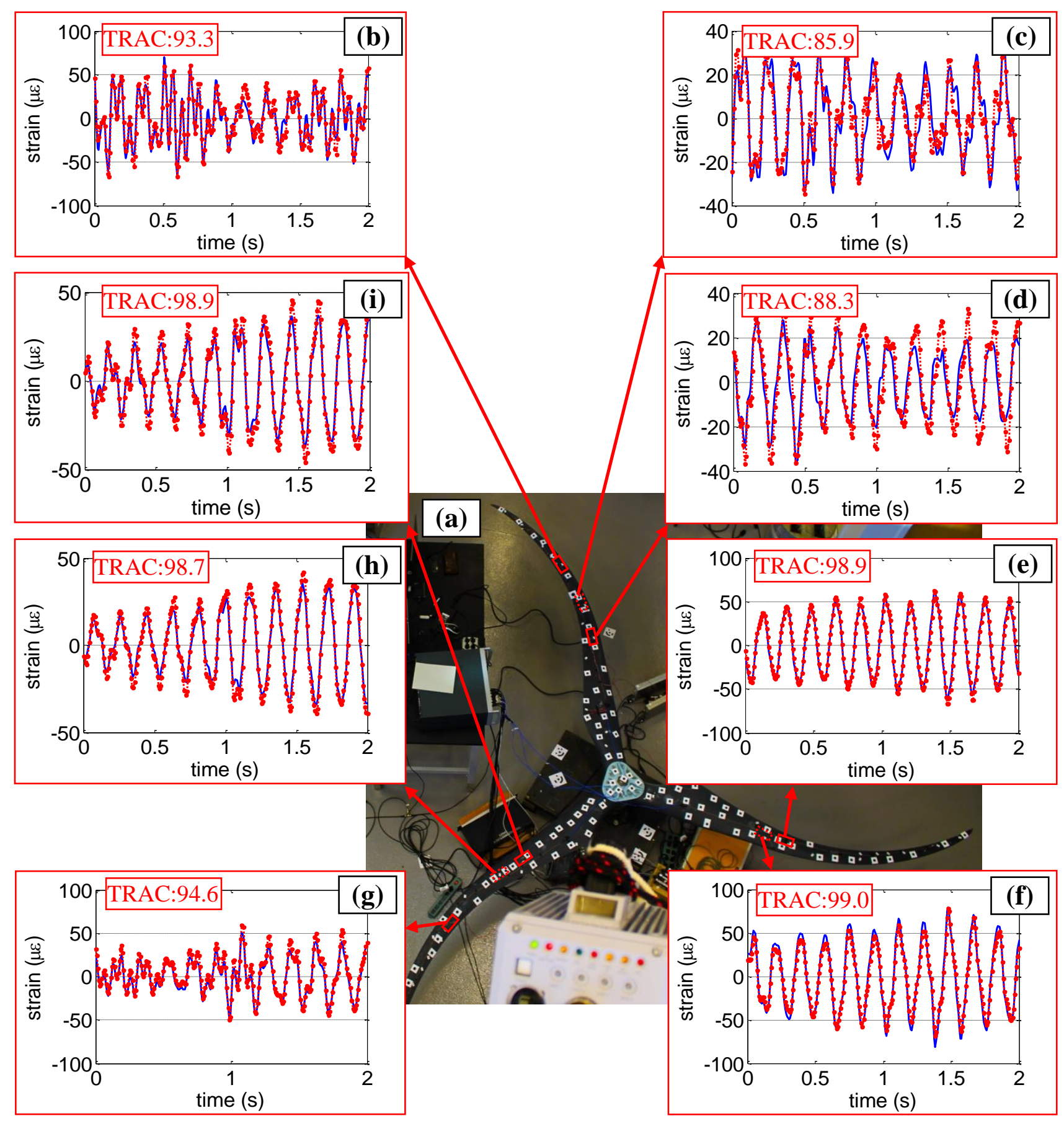

Figure 8. Comparison of the strain measured with mounted strain-gages ( $\square$ ) and the predicted strain using the proposed approach (:) when the turbine was impacted with three impact hammers simultaneously. (a) The photo shows the location of the strain-gages (red boxes), where the comparison is performed. Comparison at location of sensors (b) $S 11$, (c) $S 12$, (d) $S 13$, (e) $S 21$, (f) $S 22$, (g) $S 31$, (h) $S 32$, and (i) $S 33$. It is important to note that sensors $S 12$, S22, and $S 32$ were mounted to the underside surface of the blades where no optical targets were placed or measured. 


\subsection{Random Excitation with a Mechanical Shaker}

In the third case, the wind turbine was excited using a mechanical shaker with a random input. The auto power spectrum of the input force is shown in Figure 9. Based on the spectrum, the excitation force should excite all the modes below approximately $60 \mathrm{~Hz}$.

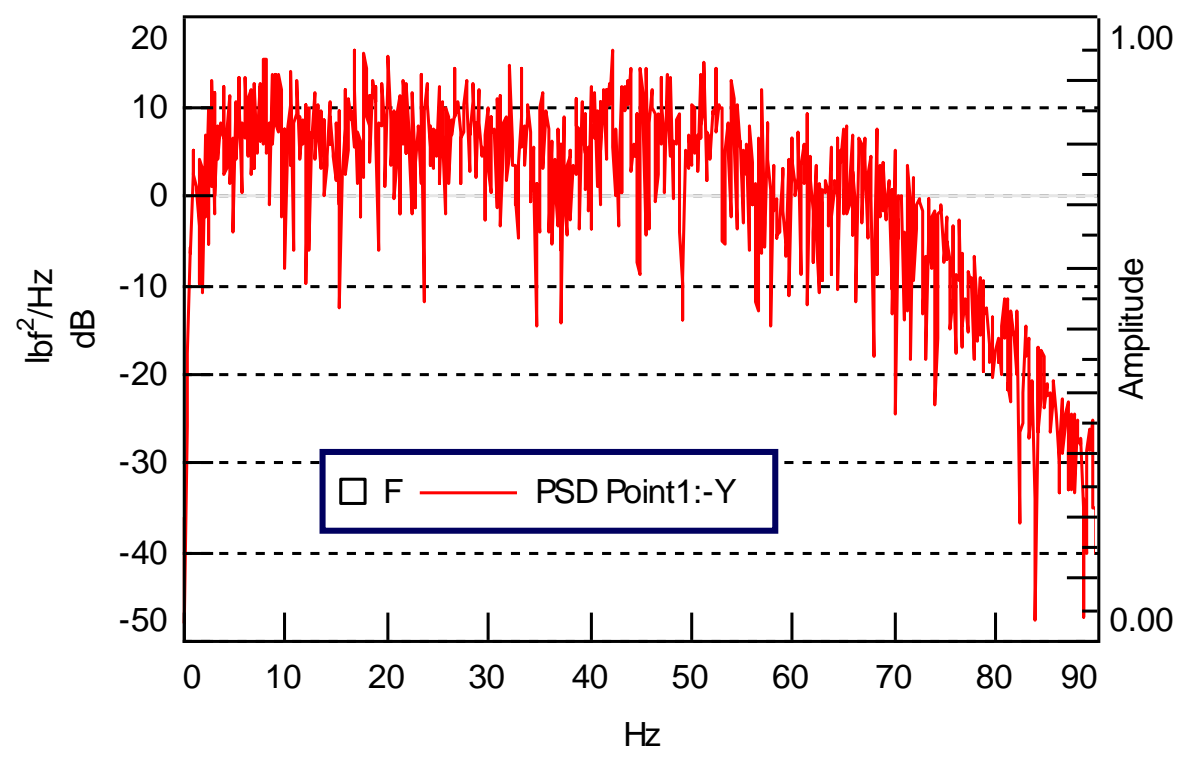

Figure 9. Reference auto-power spectrum of the random force excitation test using the shaker.

A comparison between the measured and predicted strain for the random force excitation with the shaker is shown in Figure 10. The results show very good agreement between the results for most of the strain-gages. It should be mentioned that for the strain-gages that do not have high TRAC values, the strain levels are very low and are below the noise floor of the proposed technique. In these cases there are slight variations and the predicted strain may differ from the measured strain. However, the magnitude of the predicted strain correlates still well with the measured strain. 


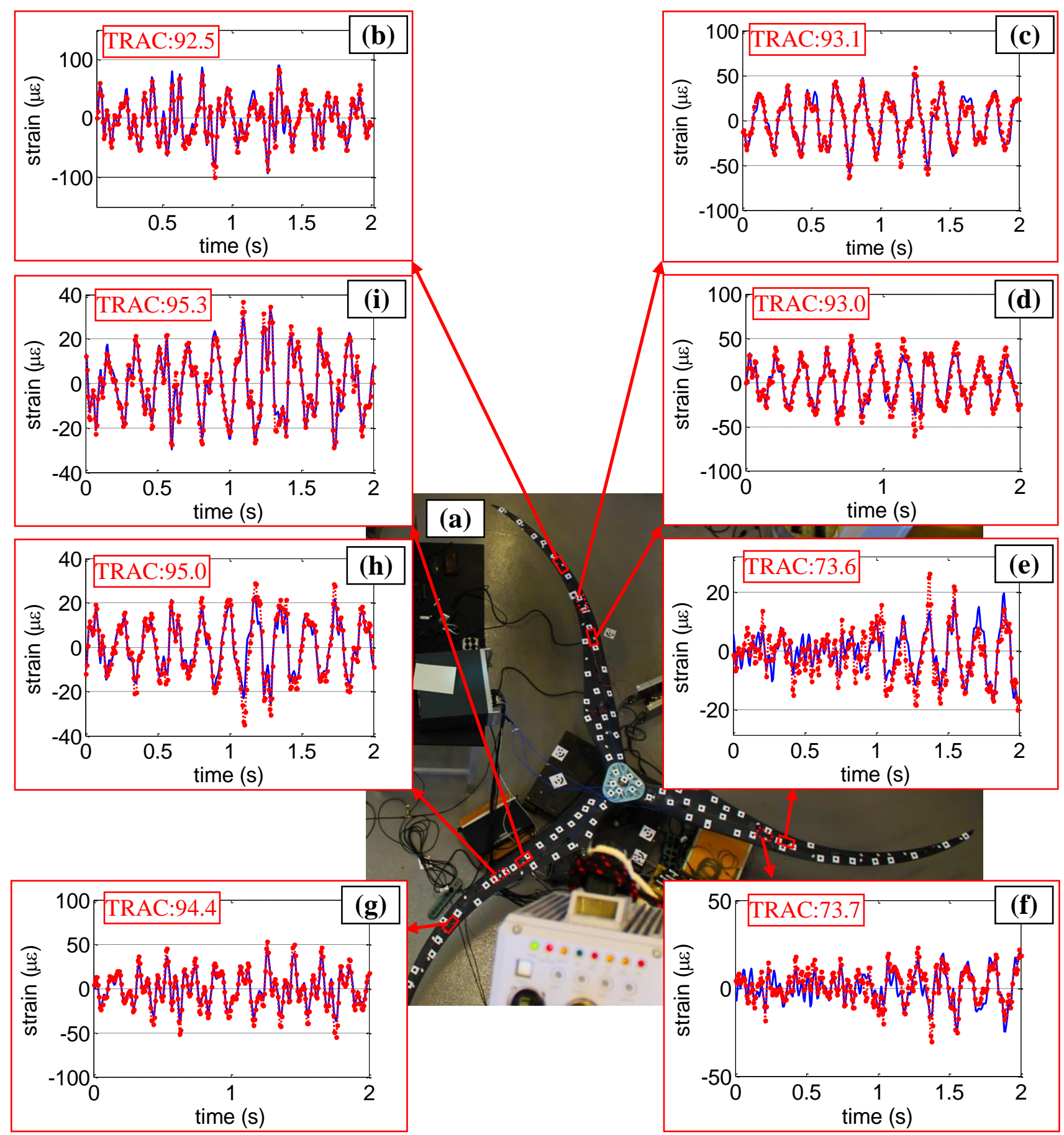

Figure 10. Comparison of the strain measured with mounted strain-gages ( $\square$ ) and the predicted strain using the proposed approach (:)when the wind turbine was excited by a shaker with a burst random excitation. (a) The photo shows the location of the strain-gages (red boxes), where the comparison is performed. Comparison at location of sensors (b) $S 11$, (c) $S 12$, (d) $S 13$, (e) $S 21$, (f) $S 22$, (g) $S 31$, (h) $S 32$, and (i) S33. It is important to note that sensors $S 12$, S22, and $S 32$ were mounted to the underside surface of the blades where no optical targets were placed or measured. 


\section{OBSERVATIONS}

Performing the technique to expand real time operating data on a structure with several components and subjected to arbitrary excitation revealed several important observations.

The number of modes that were needed to expand the real time operating data on a wind turbine depends on the modes that were excited. In this paper, the measured strain showed that most of the modes that were excited were below $60 \mathrm{~Hz}$. Therefore, all the modes below $60 \mathrm{~Hz}$ (20 modes) were used for the expansion. In the process of analyzing the number of modes required for the expansion process, it was found that in order to get good accuracy, the number of modes to be expanded must span the frequency range of the response produced by the external excitation. Using more modes than required to expand the measure displacement does not produce appreciably better results. The results of the paper revealed that although not all the FE modes had strong correlations to the experimental modes, the 20 modes that were used could effectively span the time trace of the operating data in the full-space model. Therefore, they could effectively show the dynamic strain with high accuracy.

The effects of choosing different sets of data points in SEREP to predict dynamic strain prediction for an academic structure was studied by Pingle et al. [46]. It was shown that the method is very robust and can predict the strain with very few number of measurement points. It should be mentioned that not only the number of measurement points are important but also the locations of the points play an important role in the data expansion. The minimum number of required sensors also depends on the type of the excitation and the number of modes excited during the measurement. Studying these effects is beyond the scope of the current work and will be investigated in future work.

The difference between the measured and predicted strain can be attributed to the noise floor of the optical system, the correlation between the FE and turbine modes, and the difference in sample rate of the two measurement systems.

To identify the accuracy of the technique in predicting dynamic strain, a noise floor measurement was performed on the turbine in a stationary state. In this measurement, the wind turbine was not excited by any external sources. The measured optical displacement error (noise floor) in this test was augmented using the expansion algorithm. The expanded noise floor displacement was applied to the finite element model. The results of this test showed the noise level in the predicted strain. As can be seen in Figure 11, even when the turbine is in stationary state, the predicted strain shows a variation of strain between -10 to 6 micro-strain. These results help to quantify the accuracy of the technique in predicting measured strain. As can be seen in Figure 10, the difference between measured and predicted strain is noticeable in the low level of strain, where the measured strain is close to the noise floor. 


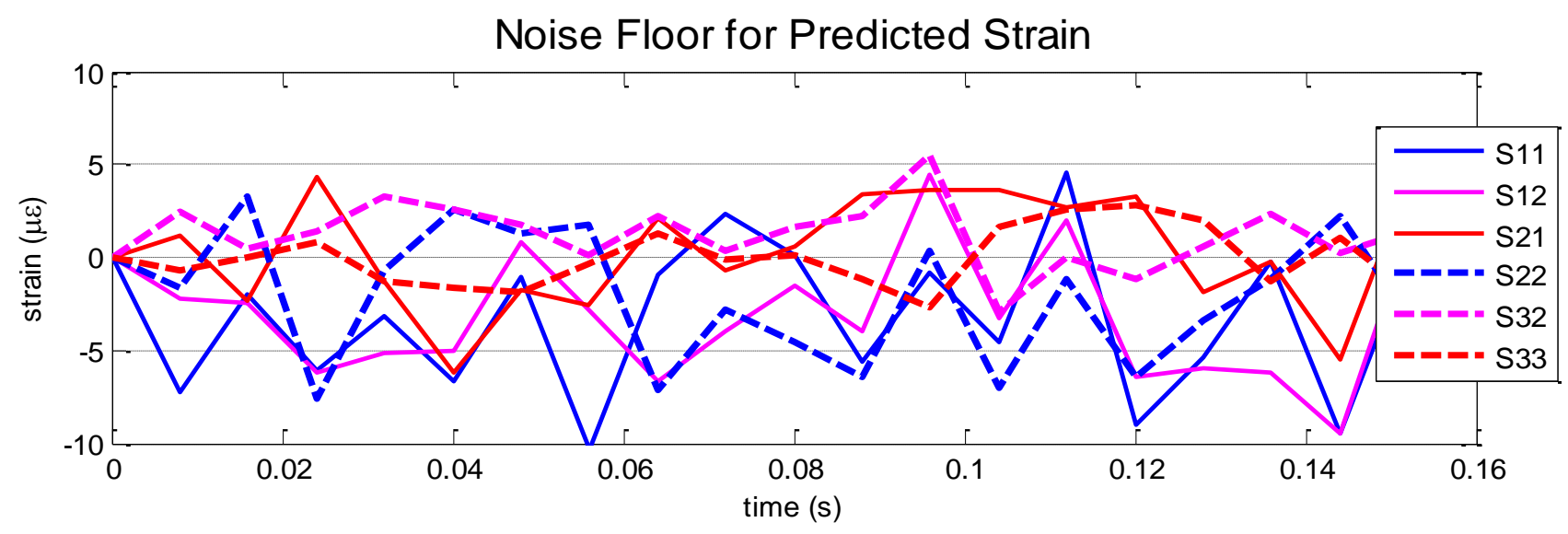

Figure 11. Noise level of the predicted strain at the locations of physical strain-gages.

Another thing that should be noted is that the cameras were capturing photos with a rate of 125 fps while strain-gages collected data at the frequency of $256 \mathrm{~Hz}$. Therefore, these two methods did not measure with equivalent sampling rates. Another possible cause of the deviation at low levels between the predicted and measured strain is the difference in sample rate. It is possible that the strain gage measurement could capture small changes in strain with higher accuracy than compared to the predicted strain.

It should also be noted that the proposed method can be used to extract dynamic strain not only on the visible surface of the structure but also on non-visible parts. For the results in Figures 6, 8, and 10, three strain-gages (S12, S22, and S32) were mounted on the underside surface of the structure; still strong correlations between the measured and predicted strain could be seen. As shown in Figure 12, the strain could also be extracted inside the blades where no-other sensor could be mounted.

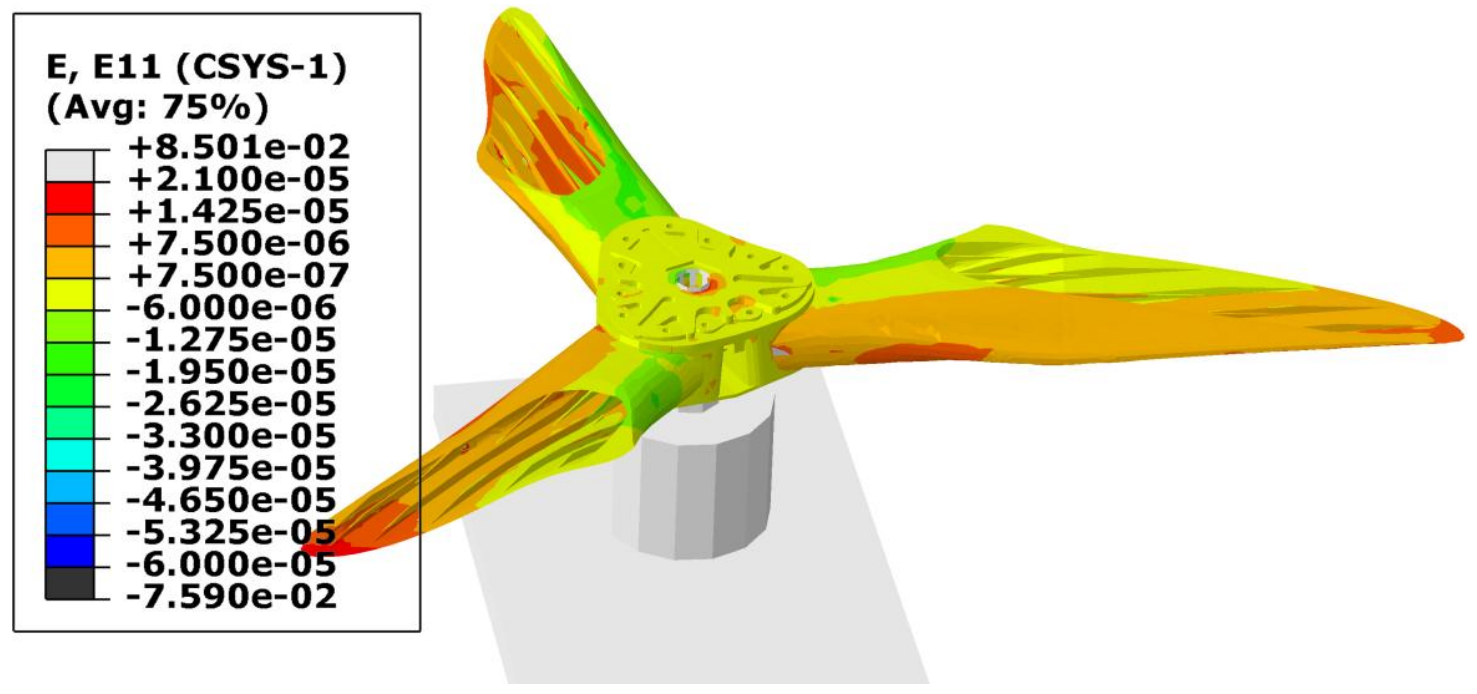


Figure 12. A section view of the turbine showing the strain distribution inside the blade at one instant of time.

The results of the paper are very encouraging with the proposed technique having a higher accuracy than compared to measuring strain by using digital image correlation. The accuracy of the digital image correlation that is frequently used for measuring full-field surface strain is reported in the literature is approximately 30100 microstrains (depending on camera resolution, facet size, facet step, computation size, and filters used). For instance, the high-speed cameras used in the current work were previously used for a DIC measurement on a small field of view $\left(\sim 1 \mathrm{~m}^{2}\right)$ of a single blade [8,9]. It was seen that the accuracy of the DIC was approximately 50-100 microstrains. In the current test, the field of view $\left(\sim 16 \mathrm{~m}^{2}\right)$ was several time larger than compared to the single blade DIC case $\left(\sim 1 \mathrm{~m}^{2}\right)$ in the previous work. Generally, it is expected that the accuracy decreases with the extending the field of view. However, the results of the paper showed that the proposed method generated strain with an accuracy up to 5 microstrain even for the large field of view. Furthermore, the technique does not need the distributed pattern surface preparation that is required for DIC measurement. This paper has demonstrated the strong potential of the proposed technique for full-field strain extraction by using displacements of optical targets.

Some of the limitations of the approach includes: 1) identifying the appropriate number of modes to include in the expansion, 2) validating that the finite element model accurately represents the dynamics of the structures, 3) using cameras with sufficient sample rate and resolution, and 4) including a sufficient number of optical targets to be used to process the data. The sensitivity of the approach as a function of these parameters is not well understood at this time and will be the focus of future studies.

\section{CONCLUSIONS}

The expansion technique was used to expand the limited set of measured displacement data obtained by using optical targets and three-dimensional point tracking. Dynamic strain on a stationary wind turbine subjected to arbitrary excitations was successfully extracted by applying the expanded displacement to the validated finite element model of the structure. The results showed excellent agreement between the strain predicted by using proposed method and the experimentally measured strain using strain-gages. The results demonstrate that the proposed technique can predict the dynamic strain on structures containing several components and subjected to complicated loading conditions with accuracy even higher than that obtained by measuring full-field strain using DIC. The results revealed that using an appropriate number of modes to expand the measured displacement is a critical part of the technique when the structure is subjected to arbitrary excitations. The technique was able to extract the strain not only on the surface of the structure but also within the interior parts of the the structure. This proposed method has tremendous potential for being applied to operating 
structures such as wind turbines and helicopter rotor blades for model validation or for structural health monitoring.

\section{ACKNOWLEDGEMENTS}

This research presented in this paper is partly supported by the National Science Foundation under Grant Number 1230884 (Achieving a Sustainable Energy Pathway for Wind Turbine Blade Manufacturing). Any opinions, findings, and conclusions or recommendations expressed in this material are those of the author(s) and do not necessarily reflect the views of the National Science Foundation.

\section{REFERENCES}

[1] M. Malkin, Reliability Trends, in: Sandia National Laboratories Wind Turbine Blade Workshop, Albuquerque, NM, 2014.

[2] P.G. Ifju, B. Stanford, M. Sytsma, R. Albertani, Analysis of a flexible wing micro air vehicle, in: 25th AIAA Aerodynamic Measurement Technology and Ground Testing Conference, San Francisco, CA, 2006. pp. 734-748

[3] T. Sibert, T. Becker, K. Spiltthof, I. Neumann, R. Krupka, High-speed digital image correlation: Error estimations and applications, Optical Engineering, 46 (2007). DOI:10.1117/1.2741217.

[4] M.N. Helfrick, C. Niezrecki, P. Avitabile, T. Schmidt, 3D digital image correlation methods for full-field vibration measurement, Mechanical Systems and Signal Processing, 25 (2011) 917-927. DOI:10.1016/j.ymssp.2010.08.013.

[5] T. Siebert, M.J. Crompton, Application of high speed digital image correlation for vibration mode shape analysis, in: SEM Annual Conference and Exposition on Experimental and Applied Mechanics 2010, Indianapolis, IN, 2010. pp. 1776-1783

[6] C. Warren, C. Niezrecki, P. Avitabile, P. Pingle, Comparison of FRF measurements and mode shapes determined using optically image based, laser, and accelerometer measurements, Mechanical Systems and Signal Processing, 25 (2011) 2191-2202. DOI:10.1016/j.ymssp.2011.01.018.

[7] J. Baqersad, J. Carr, T. Lundstrom, C. Niezrecki, P. Avitabile, M. Slattery, Dynamic characteristics of a wind turbine blade using 3D digital image correlation, in: Health Monitoring of Structural and Biological Systems 2012, San Diego, CA, 2012. DOI:10.1117/12.915377.

[8] J. Carr, J. Baqersad, C. Niezrecki, P. Avitabile, M. Slattery, Dynamic stress-strain on turbine blades using digital image correlation techniques Part 2: Dynamic measurements, in: 30th IMAC, A Conference on Structural Dynamics, 2012, Jacksonville, FL, 2012. pp. 221-226. DOI:10.1007/978-1-4614-2422-2_21.

[9] J. Carr, J. Baqersad, C. Niezrecki, P. Avitabile, M. Slattery, Dynamic stress-strain on turbine blade using digital image correlation techniques Part 1: Static load and calibration, in: 30th IMAC, A Conference on Structural Dynamics, 2012, Jacksonville, FL, 2012. pp. 215-220. DOI:10.1007/978-1-4614-24222_20.

[10] P. Poozesh, J. Baqersad, C. Niezrecki, E. Harvey, R. Yarala, Full Field Inspection of a Utility Scale Wind Turbine Blade Using Digital Image Correlation, in: CAMX, Orlando, FL, 2014.

[11] M.N. Helfrick, P. Pingle, C. Niezrecki, P. Avitabile, Optical non-contacting vibration measurement of rotating turbine blades, in: 27th Conference and Exposition on Structural Dynamics 2009, IMAC XXVII, Orlando, FL, 2009. 
[12] W. Wang, J.E. Mottershead, T. Siebert, A. Pipino, Frequency response functions of shape features from full-field vibration measurements using digital image correlation, Mechanical Systems and Signal Processing, 28 (2012) 333-347. DOI:10.1016/j.ymssp.2011.11.023.

[13] W. Wang, J.E. Mottershead, Adaptive moment descriptors for full-field strain and displacement measurements, Journal of Strain Analysis for Engineering Design, 48 (2013) 16-35

[14] W. Wang, J.E. Mottershead, A. Ihle, T. Siebert, H. Reinhard Schubach, Finite element model updating from full-field vibration measurement using digital image correlation, Journal of sound and vibration, 330 (2011) 1599-1620

[15] W. Wang, J.E. Mottershead, E. Patterson, T. Siebert, A. Ihle, Model updating using shape descriptors from full-field images, in: Conference Proceedings of the Society for Experimental Mechanics Series, 2013. pp. 425-436

[16] T. Lundstrom, J. Baqersad, C. Niezrecki, P. Avitabile, Using high-speed stereophotogrammetry techniques to extract shape information from wind turbine/rotor operating data, in: 30th IMAC, A Conference on Structural Dynamics, 2012, Jacksonville, FL, 2012. pp. 269-275. DOI:10.1007/978-14614-2419-2_26.

[17] C. Warren, C. Niezrecki, P. Avitabile, Determination of wind turbine operating deflection shapes using full-field 3D point-tracking, in: 29th IMAC, a Conference on Structural Dynamics, 2011, Jacksonville, FL, 2011. pp. 217-226

[18] M. Ozbek, D.J. Rixen, O. Erne, G. Sanow, Feasibility of monitoring large wind turbines using photogrammetry, Energy, 35 (2010) 4802-4811. DOI:10.1016/j.energy.2010.09.008.

[19] T. Lundstrom, J. Baqersad, C. Niezrecki, Using high-speed stereophotogrammetry to collect operating data on a Robinson R44 Helicopter, in: 31st International Modal Analysis Conference on Structural Dynamics, IMAC 2013, Garden Grove, CA, 2013. pp. 401-410. DOI:10.1007/978-1-4614-6546-1_44.

[20] L.E. Olson, A.I. Abrego, D.A. Barrows, A.W. Burner, Blade deflection measurements of a full-scale UH-60A rotor system, in: AHS Aeromechanics Specialists Conference 2010, San Francisco, CA, 2010. pp. 738-747

[21] O. Schneider, Analysis of SPR measurements from HART II, Aerospace Science and Technology, 9 (2005) 409-420. DOI:10.1016/j.ast.2005.01.013.

[22] O. Schneider, B.G. Van der Wall, K. Pengel, HART II Blade Motion Measured by Stereo Pattern Recognition (SPR), in: American Helicopter Society 59th Annual Forum, Phoenix, AZ, 2003.

[23] A. Tessler, J.L. Spangler, A variational principle for reconstruction of elastic deformations in shear deformable plates and shells, National Aeronautics and Space Administration, Langley Research Center, 2003

[24] A. Tessler, Structural analysis methods for structural health management of future aerospace vehicles, Key Engineering Materials, 347 (2007) 57-66

[25] A. Tessler, J.L. Spangler, A least-squares variational method for full-field reconstruction of elastic deformations in shear-deformable plates and shells, Computer Methods in Applied Mechanics and Engineering, 194 (2005) 327-339. DOI:10.1016/j.cma.2004.03.015.

[26] R.J. Guyan, Reduction of stiffness and mass matrices, AIAA journal, 3 (1965) 380-380

[27] R.L. Kidder, Reduction of structural frequency equations, AIAA journal, 11 (1973) 892-892

[28] J.C. O'Callahan, A procedure for an improved reduced system (IRS) model, in: Proceedings of the 7th International Modal Analysis Conference, Union College Press, Schenectady, NY, Las Vegas, Nevada, 1989. pp. 17-21

[29] J. O'Callahan, P. Avitabile, R. Riemer, System equivalent reduction expansion process (SEREP), in: 7th IMAC, A Conference on Structural Dynamics, 1989, 1989. pp. 29-37

[30] C. Chipman, P. Avitabile, Expansion of transient operating data, Mechanical Systems and Signal Processing, 31 (2012) 1-12. DOI:10.1016/j.ymssp.2012.04.013.

[31] P. Pingle, Prediction of Full-Field Dynamic Stress-Strain from Limited Sets of Measured Displacement Data, in: Mechanical Engineering Department, University of Massachusetts Lowell, 2010. 
[32] J. Carr, J. Baqersad, C. Niezrecki, P. Avitabile, M. Slattery, Predicting dynamic strain on wind turbine blade using digital image correlation techniques in conjunction with analytical expansion methodologies, in: 31st International Modal Analysis Conference on Structural Dynamics, IMAC 2013, Garden Grove, CA, 2013. pp. 295-302. DOI:10.1007/978-1-4614-6546-1_31.

[33] A.N. Iliopoulos, C. Devriendt, S.N. Iliopoulos, D. Van Hemelrijck, Continuous fatigue assessment of offshore wind turbines using a stress prediction technique, in: SPIE/NDE, San Diego, CA, 2014. pp. 90640S-90640S-90611

[34] P. Avitabile, S.E. Obando, K. Truong, Full Field Dynamic Deflection and Strain for Linear Components Connected with Nonlinear Connectors, in: International Conference on Noise and Vibration Engineering (ISMA2014), Leuven, Belgium, 2014.

[35] J. Harvie, S. Obando, P. Avitabile, Reduced Order System Model Nonlinear Response and Expansion for Full Field Results, in: Eleventh International Conference on Recent Advances in Structural Dynamics,, Pisa, Italy, 2013.

[36] P.B. Bogert, E. Haugse, R.E. Gehrki, Structural shape identification from experimental strains using a modal transformation technique, in: 44th AIAA/ASME/ASCE/AHS/ASC Structures, Structural Dynamics, and Materials Conference, 2003.

[37] A. Derkevorkian, S.F. Masri, J. Alvarenga, H. Boussalis, J. Bakalyar, W. Lance Richards, Strain-based deformation shape-estimation algorithm for control and monitoring applications, AIAA journal, 51 (2013) 2231-2240. DOI:10.2514/1.10275.

[38] M. Chierichetti, M. Ruzzene, Dynamic displacement field reconstruction through a limited set of measurements: Application to plates, Journal of sound and vibration, 331 (2012) 4713-4728

[39] M. Chierichetti, C. McColl, D. Palmer, M. Ruzzene, O. Bauchau, Combined analytical and experimental approaches to rotor components stress predictions, Proceedings of the Institution of Mechanical Engineers, Part K: Journal of Multi-body Dynamics, 225 (2011) 322-330

[40] M. Chierichetti, Load and response identification for a nonlinear flexible structure subject to harmonic loads, Journal of Computational and Nonlinear Dynamics, 9 (2014)

[41] M. Chierichetti, C. Grappasonni, G. Coppotelli, C. McColl, A modal approach for dynamic response monitoring from experimental data, Mechanical Systems and Signal Processing, 48 (2014) 199-217

[42] R.J. Allemang, D.L. Brown, Correlation coefficient for modal vector analysis, in: Proceedings of the 1st International Modal Analysis Conference \& Exhibit., Union Coll, Orlando, FL, USA, 1982. pp. 110116

[43] PONTOS v6.3, in, GOM mbH, Braunschweig, Germany 2011.

[44] J. Brown, Characterization of MSC/NASTRAN \& MSC/ABAQUS Elements for Turbine Engine Blade Frequency Analysis, in: Proc. MSC Aerospace Users' Conference Proc. MSC Aerospace Users' Conference, 1997.

[45] J. Baqersad, P. Poozesh, C. Niezrecki, P. Avitabile, Comparison of Modal Parameters Extracted Using MIMO, SIMO, and Impact Hammer Tests on a Three-Bladed Wind Turbine, in: 32th IMAC, A Conference on Structural Dynamics, Orlando, FL, 2014. DOI:10.1007/978-3-319-04774-4_19.

[46] P. Pingle, P. Avitabile, Full field dynamic stress/strain from limited sets of measured data, in: 29th IMAC, a Conference on Structural Dynamics, 2011, Jacksonville, FL, 2011. pp. 187-200 